\title{
EDITORIAL AND COMMENT The First Step is the Hardest: Overcoming Barriers to Primary Care
}

\author{
Margot Kushel, MD \\ Division of General Internal Medicine, UCSF/San Francisco General Hospital, San Francisco, CA, USA.
}

J Gen Intern Med 30(7):868-9

DOI: $10.1007 / \mathrm{s} 11606-015-3279-5$

(C) Society of General Internal Medicine 2015

I n 2007, the Institute for Healthcare Improvement developed the Triple Aim framework to describe an approach to optimizing health delivery systems. ${ }^{1}$ The Triple Aim argues that health systems must improve patient experience and population health, while reducing the cost of healthcare. To improve population health, health institutions must look beyond the walls of the health system to reach members of the targeted population who are not receiving care. This objective presents many challenges for clinicians and health systems, particularly when the targeted population is homeless. In this issue of JGIM, O'Toole et al. ${ }^{2}$ describe the results of a two-by-two randomized control trial that aimed to engage out-of-care homeless veterans into primary care. They found that two low-intensity interventions designed to overcome common reasons to not seek care increased the probability of homeless veterans visiting primary care within 4 weeks.

Homeless individuals have poor access to and use of primary care services. ${ }^{3}$ Individuals experiencing homelessness face numerous barriers to receiving primary care, ${ }^{4}$ including the lack of insurance or adequate funds to afford copayments, lack of transportation, and difficulty making (or receiving information about) appointments. Those experiencing homelessness (particularly those with co-occurring mental health or substance use disorders) face stigma that reduces their engagement with care. ${ }^{5}$ In addition to these barriers, people experiencing homelessness may, with good reason, prioritize immediate needs (shelter, food) over seeking primary care. The changes brought on by the Affordable Care Act (ACA) (in the 29 states that have enacted Medicaid expansion) hold promise to eliminate lack of insurance as a barrier to seeking care. However, the other barriers remain.

Disproportionately high rates of homelessness among veterans have led the Department of Veterans Affairs (VA) to be at the forefront of initiatives to address these barriers. In an effort to end homelessness among veterans by the end of 2015 , the VA has focused efforts on providing chronically homeless veterans with permanent supportive housing, rapidly rehousing those with recent homelessness, preventing homelessness in those at risk, and developing new models of primary

Published online March 19, 2015 care for veterans via Homeless Patient Aligned Care Teams (H-PACTs). ${ }^{6,7}$ H-PACTS, which combine features of healthcare for the homeless primary care clinics with primary care medical homes, focus on the coordination of primary care services with housing services for homeless veterans. HPACTS are staffed by multidisciplinary teams who are charged with providing accessible "just-in-time" primary care, developing trust between provider and client, and providing integrated care that provides access to the VA housing services.

While the receipt of primary care is not sufficient to ensure the health of homeless individuals, it is necessary, particularly for the vast majority of those who have chronic health conditions. The homeless population is aging faster than the general population; the median age in the United States is approximately $50 .^{8}$ The changing age structure of the homeless population increases the relevance of primary care. When this population was younger, health-sustaining efforts focused on providing appropriate care for acute illnesses (including infectious diseases), injuries, and mental health and substance abuse treatment. ${ }^{9}$ With the aging of the homeless population, there is a need to focus on managing chronic conditions. This, alongside increased options for managing mental health and substance abuse disorders in primary care, ${ }^{10}$ and the rise of co-locating other key resources in primary care (medical legal partnerships, social work services), has raised the importance of engaging homeless individuals in primary care. The VA has responded by organizing H-PACTS to provide the full panoply of health services that homeless veterans require. ${ }^{6}$ However, H-PACTS and other homelessfocused primary care sites will improve health only if they are accessed. Building high-functioning clinics is not enough: those clinics must reach their target population.

The intervention studied by O'Toole et al. did not target the highest-risk veterans who were eligible for intensive outreach services. Rather, the intervention worked to increase attendance among veterans who could benefit from primary care, but did not meet criteria for the most intensive programs. The authors hypothesized that by getting people through the door once, they could start the process of engagement into longitudinal care. The intervention focused on veterans' own motivations for care by helping them identify their need for care. It also worked to demystify the process of receiving primary care by introducing them to members of the clinic team and explaining how clinics worked, and to alleviate concerns 
about costs for services by letting veterans know that these services were free of charge. Finally, the interventions gave the veterans a taste of the range of services that were offered at the H-PACTS in order to motivate the out-of-care veterans to take the first step towards receiving care. In doing so, the intervention increased potential patients' own motivation and dispelled concerns about presenting for care.

The study compared two interventions, either alone or together, versus usual care. The interventions were designed to be low-intensity, and thus scalable. The first consisted of a 30-minute health screen performed by a registered nurse, who then used those results to engage in motivational interviewing on the importance of primary care. The other intervention involved transportation to a brief on-site clinic orientation, wherein the previously unengaged individual was introduced to members of the clinic team and given information on how to make appointments and contact the clinic. Both interventions improved clinic attendance at 4 weeks (versus usual control). Those who received the clinic orientation alone had a larger increase in first visits than those who received the nurse interview, but those who received both had the highest rates of clinic attendance, with $77.3 \%$ attending at 4 weeks and $88.7 \%$ after 6 months. Among those who made the first appointment, there were no differences in number of clinic visits or use of specialty care in the next 6 months. It is telling that those who remained in usual care were unlikely to seek care over the 6-month study period: only $37.1 \%$ of those in the usual care group had attended even one primary care visit at 6 months.

The results are impressive, but the participating patients and clinic had several advantages not shared by most homeless individuals. As the intervention targeted homeless veterans, no participants had financial barriers to clinic visits. No one had to pay either copayments or other visit-related costs for their care. Both H-PACT clinics offered open-access appointments, whereby every patient who showed up for a clinic session would be guaranteed an appointment on the same day. Because the study was conducted in urban areas, participants were all within potentially walk-able distances to the clinic and had access to public transportation. The implementation of the ACA and the expansion of primary care medical homes may move other clinic settings closer to those including in the study, but the study sites should be considered as ideal conditions. The effectiveness in non-VA and non-urban settings will likely be lower.

Despite these limitations, the study does serve as a proof of concept that relatively low-intensity interventions could increase the proportion of out-of-care individuals who present for primary care, when primary care is designed with the needs of homeless individuals in mind. The authors' focus on out-ofcare individuals takes the literature beyond where the majority of primary care research focuses - maximizing the effectiveness of care for those already in care. The authors should be applauded for expanding the notion of the intractability of barriers to accessing care in homeless individuals. By sending primary care workers to meet potential patients where they are, the investigators found that the barriers to engagement were not insurmountable. Their findings make clear that relatively low-cost, well-thought-out interventions that ask little of the participants can get participants over the initial threshold of coming in for care. Different interventions may be necessary to keep them there. With the promise of the ACA, rising rates of older people experiencing homelessness, and a renewed focus on the importance of the primary care medical home, the study by O'Toole et al. study makes an essential contribution.

Conflict of Interest: Dr. Kushel reports no conflicts of interest.

Corresponding Author: Margot Kushel, MD; Division of General Internal Medicine UCSF/San Francisco General Hospital, San Francisco, CA, USA (e-mail: Margot.kushel@ucsf.edu).

\section{REFERENCES}

1. IHI Triple Aim Initiative. Better Care for Individuals, Better Health for Populations, and Lower Per Capita Costs. Cambridge, MA: Institute for Healthcare Improvement; 2015.

2. O'Toole TP, Johnson EE, Borgia ML, Rose J. Tailoring outreach efforts to increase primary care use among homeless veterans: results of a randomized control trial. J Gen Int Med. 2015. doi:10.1007/s11606-015-3193-x.

3. Kushel MB, Vittinghoff E, Haas JS. Factors associated with the health care utilization of homeless persons. JAMA. 2001;285(2):200-206.

4. White BM, Newman SD. Access to primary care services among the homeless: a synthesis of the literature using the equity of access to medical care framework. J Prim Care Commun Health. 2015;6(2):77-87.

5. Phelan J, Link BG, Moore RE, Stueve A. The stigma of homelessness: the impact of the label "homeless" on attitudes toward poor persons. Soc Psychol Q. 1997;60(4):323-337.

6. Bamberger J. VA's HPACT Program Collaborates to Meet Healthcare, Housing Needs. Washington, DC: U.S. Interagency Council on Homelessness. Available at http://usich.gov/population/veterans/veterans_homelessness_in_focus/hpact. Accessed February 19, 2015.

7. Homeless. 2015; http://www.va.gov/homeless/. Accessed February 23, 2015.

8. Culhane DP, Metraux S, Bainbridge J. The age structure of contemporary homelessness: risk period or cohort effect? Penn School of Social Policy and Practice Working Paper; 2010.

9. Garibaldi B, Conde-Martel A, O'Toole TP. Self-reported comorbidities, perceived needs, and sources for usual care for older and younger homeless adults. J Gen Intern Med. 2005;20(8):726-730.

10. Butler M, Kane RL, McAlpine D, et al. Integration of mental health/ substance abuse and primary care. Evid Rep Technol Assess. 2008;173:1-362. 